\title{
Strong approximation of stochastic differential equations driven by a time-changed Brownian motion with time-space-dependent coefficients
}

\author{
Sixian Jin* $^{*}$ and Kei Kobayashi ${ }^{\dagger}$
}

\begin{abstract}
The rate of strong convergence is investigated for an approximation scheme for a class of stochastic differential equations driven by a time-changed Brownian motion, where the random time changes $\left(E_{t}\right)_{t \geq 0}$ considered include the inverses of stable and tempered stable subordinators as well as their mixtures. Unlike those in the work of Jum and Kobayashi (2016), the coefficients of the stochastic differential equations discussed in this paper depend on the regular time variable $t$ rather than the time change $E_{t}$. This alteration makes it difficult to apply the method used in that paper. To overcome this difficulty, we utilize a Gronwall-type inequality involving a stochastic driver to control the moment of the error process. Moreover, in order to guarantee that an ultimately derived error bound is finite, we establish a useful criterion for the existence of exponential moments of powers of the random time change.
\end{abstract}

Key words: stochastic differential equation, numerical approximation, rate of convergence, inverse subordinator, random time change, time-changed Brownian motion.

2010 Mathematics Subject Classification: 65C30, 60H10.

\section{Introduction}

Let $B=\left(B_{t}\right)_{t \geq 0}$ be a standard Brownian motion and $E=\left(E_{t}\right)_{t \geq 0}$ be a stochastic process defined by the inverse of a subordinator $D=\left(D_{t}\right)_{t \geq 0}$ with infinite Lévy measure, independent of $B$. The composition $B \circ E=\left(B_{E_{t}}\right)_{t \geq 0}$, called a time-changed Brownian motion, and its various generalizations have been widely used to model anomalous diffusions arising in e.g. physics [27, 31, 40], finance [6, 22], hydrology [1], and cell biology [35]. See Chapter 1 of [37] for details. The time-changed Brownian motion is non-Markovian $([25,26])$. Also, the identity $\mathbb{E}\left[B_{E_{t}}^{2}\right]=\mathbb{E}\left[E_{t}\right]$ holds, and in particular, if the subordinator $D$ is stable with index $\beta \in(0,1)$, then $\mathbb{E}\left[B_{E_{t}}^{2}\right]=t^{\beta} / \Gamma(1+\beta)$, which shows that in large time scales particles represented by $B \circ E$ spread at a slower rate than the rate at which Brownian particles diffuse. Moreover, the densities of $B \circ E$ satisfy the time-fractional order Fokker-Planck (or forward Kolmogorov) equation $\partial_{t}^{\beta} u(t, x)=(1 / 2) \Delta u(t, x)$, where $\partial_{t}^{\beta}$ denotes the Caputo fractional derivative of order $\beta$ with respect to the variable $t$. Various extensions of $B \circ E$ and their associated fractional order Fokker-Planck equations have been investigated, including time-changed fractional Brownian motions (see $[7,8,24]$ ) and stochastic differential equations (SDEs) involving the random time change $E$ (see below).

In this paper, we investigate the rate of strong convergence of a numerical approximation scheme for an SDE of the form

$$
X_{t}=x_{0}+\int_{0}^{t} F\left(s, X_{s}\right) \mathrm{d} E_{s}+\int_{0}^{t} G\left(s, X_{s}\right) \mathrm{d} B_{E_{s}},
$$

\footnotetext{
*Fordham University. Email: sjin27@fordham.edu

${ }^{\dagger}$ Corresponding author. Fordham University. Email: kkobayashi5@fordham.edu
} 
where the coefficients $F$ and $G$ satisfy some regularity conditions. Note that since $E$ is a nondecreasing process and $B \circ E$ is a martingale with respect to a certain filtration, SDE (1) is understood within the framework of stochastic integrals driven by semimartingales; see [17] or the beginning of Section 3 for details. SDE (1) and its extensions to cases involving jump components have recently drawn more and more attention. For example, papers [29, 30, 39] established stability in various senses of solutions of SDEs driven by time-changed processes using the time-changed Itô formula derived in [17] and its generalizations. In [23, 28], fractional order Fokker-Planck equations were derived for solutions of SDEs of the form (1) with Lévy noise terms added. Practical situations where such SDEs naturally arise include Langevin-type subdiffusive dynamics in physics with force terms of the form $F(t, x)=f_{1}(t) f_{2}(x)$ and constant diffusion coefficient $[10,38]$ and stock price dynamics with trapping events and volatility clustering in finance where the diffusion coefficient may also take the form $G(t, x)=g_{1}(t) g_{2}(x)[18,21,32]$. As the well-established theory of classical Itô SDEs (without a random time change) enabled a number of mathematicians and scientists in various fields to explore questions about more complicated diffusion processes than the Brownian motion itself, further investigations of SDE (1) and its extensions are necessary and expected in order to deal with more complex anomalous diffusions.

This paper partly builds upon some results established in [19,20], which investigated the process $X$ defined by (1) with $F(t, x)=F(t)$ having finite variation and $G(t, x) \equiv 1$ (in which case (1) is no longer an SDE since the coefficients do not depend on $x$ ). In those papers, a numerical approximation scheme for $X$ was presented together with the rate of strong convergence. On the other hand, even though the idea employed in $[19,20]$ together with the Euler-Maruyama scheme allows us to approximate the solution $X$ of SDE (1) with general space-time-dependent coefficients, convergence of the approximation scheme has not been investigated. Establishing the rate of convergence for the scheme is an extremely important issue both theoretically and practically in numerical analysis of complex systems displaying anomalous dynamics, and that is the main contribution of this paper. In particular, our convergence results will help justify the use of Monte Carlo techniques in approximating the solutions of the fractional order Fokker-Planck equations derived in [23, 28].

The main difficulty in analyzing SDE (1) lies in the "asynchrony" between the time variable $s$ in the integrands and the time change $E_{s}$ in the driving processes. To see this, consider instead an SDE with a synchronized time clock of the form

$$
X_{t}=x_{0}+\int_{0}^{t} F\left(E_{s}, X_{s}\right) \mathrm{d} E_{s}+\int_{0}^{t} G\left(E_{s}, X_{s}\right) \mathrm{d} B_{E_{s}},
$$

where the coefficients depend on $E_{s}$ rather than $s$. For this SDE, the associated fractional order Fokker-Planck equation was established in [9], and the orders of strong and weak convergence of an approximation scheme were derived in [14]. The key fact used in those papers was the duality principle between SDE (2) and the classical Itô SDE

$$
Y_{t}=x_{0}+\int_{0}^{t} F\left(s, Y_{s}\right) \mathrm{d} s+\int_{0}^{t} G\left(s, Y_{s}\right) \mathrm{d} B_{s} .
$$

Namely, if $Y_{t}$ solves (3), then $X_{t}:=Y_{E_{t}}$ solves (2), while if $X_{t}$ solves (2), then $Y_{t}:=X_{D_{t}}$ solves (3), where $D$ is the original subordinator (see Theorem 4.2 of [17]). This one-to-one correspondence between the two SDEs allows us to approximate the solution of (2) by the composition $X_{t}^{\delta}:=\left(Y^{\delta} \circ E^{\delta}\right)_{t}=Y_{E_{t}^{\delta}}^{\delta}$, where $\delta \in(0,1)$ refers to an equidistant step size, $E^{\delta}$ is the approximation process for $E$ defined in $[19,20]$, and $Y^{\delta}$ is the approximation of $Y$ based on the Euler-Maruyama scheme. The independence assumption between $B$ and $E$ together with representation (3) implies independence between $Y$ and $E$, and therefore, the two approximation processes $Y^{\delta}$ and $E^{\delta}$ can be constructed independently and simply composed to define the approximation process $X^{\delta}$. The independence also allows the two types of errors (one ascribed to the approximation of $Y$ and the other due to the approximation of $E$ ) to be analyzed separately.

On the other hand, a referee of the paper [14] raised an important question of whether the methods used for SDE (2) can be applied to SDE (1) or not. Unfortunately, the approach used in [14] no longer works for approximation of the solution of SDE (1). Indeed, the duality principle implies the 
corresponding SDE takes the form

$$
Y_{t}=x_{0}+\int_{0}^{t} F\left(D_{s-}, Y_{s}\right) \mathrm{d} s+\int_{0}^{t} G\left(D_{s-}, Y_{s}\right) \mathrm{d} B_{s}
$$

which clearly shows $Y$ depends on $D$ (and hence on $E$ as well), and consequently, the conditioning argument based on the independence of $Y$ and $E$ used for SDE (2) cannot be applied. This observation, which appears in Remark 3.2(5) of [14], forces us to take a different approach in dealing with SDE (1). In particular, the duality principle is not used at all. Instead, we utilize a Gronwall-type inequality involving a stochastic driver to control the moment of the error process. Moreover, in order to eventually obtain a meaningful bound for the moment in Section 3, we derive a useful criterion for the existence of the exponential moment $\mathbb{E}\left[e^{\lambda E_{t}^{r}}\right]$ of the $r$ th power of the inverse subordinator in Section 2; this may be of independent interest to some readers. It is also worth mentioning that, even though the approximation scheme used in this paper is of Euler-Maruyama type, the order of strong uniform convergence to be established in Theorem 7 is strictly less than $1 / 2$. This is different from the classical setting of Itô SDEs without a random time change, where the order $1 / 2$ can be achieved. An explanation of why this phenomenon occurs under the time change will be discussed in Remark 9(3).

\section{Exponential moments of powers of inverse subordinators}

Throughout the paper, $(\Omega, \mathcal{F}, \mathbb{P})$ denotes a complete probability space and $D=\left(D_{t}\right)_{t \geq 0}$ denotes a subordinator starting at 0 with Laplace exponent $\psi$ with killing rate 0 , drift 0 , and Lévy measure $\nu$; i.e. $D$ is a one-dimensional nondecreasing Lévy process with càdlàg paths starting at 0 with Laplace transform

$$
\mathbb{E}\left[e^{-s D_{t}}\right]=e^{-t \psi(s)}, \quad \text { where } \psi(s)=\int_{0}^{\infty}\left(1-e^{-s y}\right) \nu(\mathrm{d} y), \quad s>0,
$$

with $\int_{0}^{\infty}(y \wedge 1) \nu(\mathrm{d} y)<\infty$. We focus on the case when the Lévy measure $\nu$ is infinite (i.e. $\left.\nu(0, \infty)=\infty\right)$, which implies compound Poisson subordinators are excluded from our discussion. Let $E=\left(E_{t}\right)_{t \geq 0}$ be the inverse of $D$; i.e.

$$
E_{t}:=\inf \left\{u>0 ; D_{u}>t\right\}, \quad t \geq 0 .
$$

We call $E$ an inverse subordinator. The assumption that $\nu(0, \infty)=\infty$ implies that $D$ has strictly increasing paths with infinitely many jumps (see e.g. [34]), and therefore, $E$ has continuous, nondecreasing paths starting at 0 . If the subordinator $D$ is stable with index $\beta \in(0,1)$, then $\psi(s)=s^{\beta}$ and the corresponding time change $E$ is called an inverse $\beta$-stable subordinator. Note that the jumps of $D$ correspond to the (random) time intervals on which $E$ is constant, and during those constant periods, any time-changed process of the form $X \circ E=\left(X_{E_{t}}\right)_{t \geq 0}$ also remains constant. If $B$ is a standard Brownian motion independent of $D$, we can regard particles represented by the time-changed Brownian motion $B \circ E$ as being trapped and immobile during the constant periods. See Figure 1.

Any inverse subordinator $E$ with infinite Lévy measure is known to have the exponential moment; i.e. $\mathbb{E}\left[e^{\lambda E_{t}}\right]<\infty$ for all $\lambda>0$ and $t>0$ (see [14, 22]). However, as is shown in Theorem 1, whether the expectation $\mathbb{E}\left[e^{\lambda E_{t}^{r}}\right]$ with $r>1$ exists or not depends on the nature of the time change. In particular, if $E$ is an inverse $\beta$-stable subordinator, then $\mathbb{E}\left[e^{\lambda E_{t}^{2}}\right]$ exists if $1 / 2<\beta<1$ while it does not if $0<\beta<1 / 2$. When $\beta=1 / 2$, whether the expectation exists or not depends on the relationship between $\lambda$ and $t$; see Remark 6(2).

One situation where the need for the information about the existence of $\mathbb{E}\left[e^{\lambda E_{t}^{r}}\right]$ arises is implicitly discussed in [14]. Namely, consider a sequence $\left\{X^{(n)}\right\}_{n \geq 1}$ of stochastic processes converging to $X$ in $L^{p}$ with $p \geq 1$ uniformly on compact subsets of $[0, \infty)$ with a bound $\left\|\sup _{s \in[0, t]}\left|X_{s}^{(n)}-X_{s}\right|\right\|_{L^{p}(\Omega)} \leq a_{n} g(t)$ for all $t>0$, where $\left\{a_{n}\right\}$ is a sequence approaching 0 and $g(t)$ is a function of $t$. If $E$ is an inverse subordinator independent of both $X$ and $\left\{X^{(n)}\right\}$, then a simple conditioning argument yields

$$
\left\|\sup _{s \in[0, t]}\left|X_{E_{s}}^{(n)}-X_{E_{s}}\right|\right\|_{L^{p}(\Omega)}=\left\|\sup _{s \in\left[0, E_{t}\right]}\left|X_{s}^{(n)}-X_{s}\right|\right\|_{L^{p}(\Omega)} \leq a_{n}\left\|g\left(E_{t}\right)\right\|_{L^{p}(\Omega)} .
$$




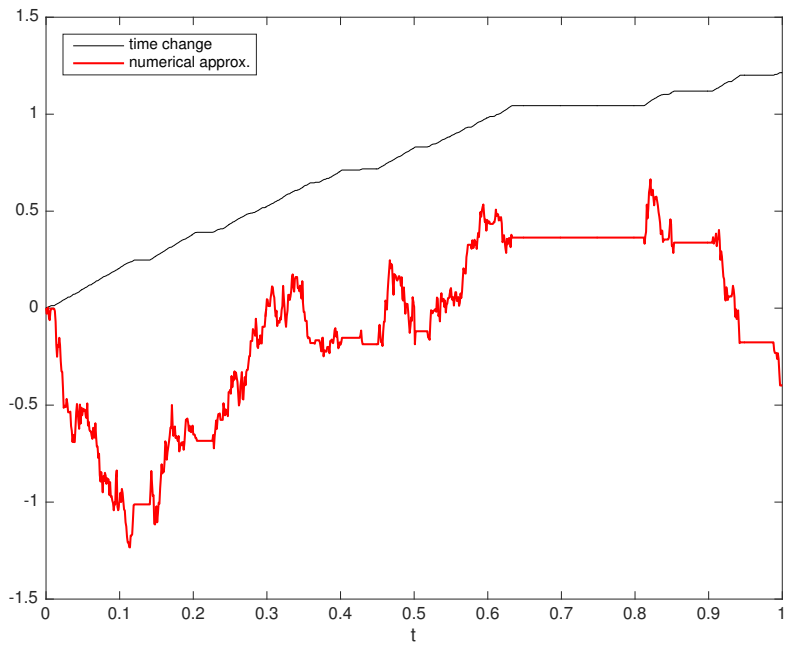

Figure 1: Sample paths of an inverse 0.9-stable subordinator $E$ (black) and the corresponding timechanged Brownian motion $B \circ E$ (red), which share the same constant periods.

Therefore, if e.g. $g(t)$ takes the form $g(t)=c e^{c t^{2}}$ and $E$ is an inverse $\beta$-stable subordinator with $\beta \in(0,1 / 2)$, then by the discussion in the previous paragraph, $\left\|g\left(E_{t}\right)\right\|_{L^{p}(\Omega)}=c\left(\mathbb{E}\left[e^{p c E_{t}^{2}}\right]\right)^{1 / p}=\infty$, and consequently, the above bound is no longer meaningful. Such a simple example illustrates the significance of criteria for the existence and non-existence of the expectation of the form $\mathbb{E}\left[e^{\lambda E_{t}^{r}}\right]$.

To describe the kinds of inverse subordinators we are mainly concerned with in this paper, let us introduce the notion of regularly varying and slowly varying functions. A function $f:(0, \infty) \rightarrow(0, \infty)$ is said to be regularly varying at $\infty$ with index $\alpha \in \mathbb{R}$ if $\lim _{s \rightarrow \infty} f(c s) / f(s)=c^{\alpha}$ for any $c>0$. We denote by $\mathrm{RV}_{\alpha}$ the class of regularly varying functions at $\infty$ with index $\alpha$. A function $\ell:(0, \infty) \rightarrow(0, \infty)$ is said to be slowly varying at $\infty$ if $\ell \in \mathrm{RV}_{0}$ (i.e. $\ell \in \mathrm{RV}_{\alpha}$ with $\alpha=0$ ). Every regularly varying function $f$ with index $\alpha \in \mathbb{R}$ is represented as $f(s)=s^{\alpha} \ell(s)$ with $\ell$ being a slowly varying function.

Note that the following two Laplace exponents are regularly varying at $\infty$ with index $\beta \in(0,1)$ : $\psi(s)=s^{\beta}$, which corresponds to a stable subordinator with index $\beta$, and $\psi(s)=(s+\kappa)^{\beta}-\kappa^{\beta}$ with $\kappa>0$, which corresponds to an exponentially tempered (or tilted) stable subordinator with index $\beta$ and tempering factor $\kappa$. On the other hand, $\psi(s)=\log (1+s)$, which corresponds to a Gamma subordinator, is slowly varying at $\infty$. We now state the main theorem of this section, which will be used in the proof of Theorem 7 in the next section.

Theorem 1. Let $E$ be the inverse of a subordinator $D$ whose Laplace exponent $\psi$ is regularly varying at $\infty$ with index $\beta \in[0,1)$. If $\beta=0$, assume further that $\nu(0, \infty)=\infty$. Fix $\lambda>0, t>0$ and $r>0$.

(1) If $r<1 /(1-\beta)$, then $\mathbb{E}\left[e^{\lambda E_{t}^{r}}\right]<\infty$.

(2) If $r>1 /(1-\beta)$, then $\mathbb{E}\left[e^{\lambda E_{t}^{r}}\right]=\infty$.

To establish Theorem 1, we will use tail probability estimates for subordinators given in [13]. The estimates are given in terms of the Laplace exponent $\psi$ and hence easily applicable to quite general situations. Let us introduce some auxiliary notations used in Section 5 of [13]. For a subordinator $D$ with Laplace exponent $\psi$ in (5) and infinite Lévy measure (i.e. $\nu(0, \infty)=\infty$ ), let

$$
g(s):=\psi^{\prime}(s), \quad R(s):=\psi(s)-s \psi^{\prime}(s), \quad s>0 .
$$

Note that $\psi$ is a Bernstein function defined on $(0, \infty)$; i.e. $\psi \in C^{\infty}(0, \infty)$ with $\psi>0$ and $(-1)^{n} \psi^{(n)}<0$ for all $n \in \mathbb{N}$. This particularly implies that $g$ is a continuous, strictly decreasing function with $g(0)=$ $\int_{0}^{\infty} y \nu(\mathrm{d} y)$ and $g(\infty)=0$, while $R$ is a continuous, strictly increasing function with $R(0)=0$ and 
$R(\infty)=\nu(0, \infty)=\infty$, where we follow the convention $f(0):=\lim _{s \rightarrow 0} f(s)$ and $f(\infty):=\lim _{s \rightarrow \infty} f(s)$ for a given function $f$ defined on $(0, \infty)$. The condition $\nu(0, \infty)=\infty$ guarantees that the inverse $E$ of $D$ has a finite exponential moment; i.e. $\mathbb{E}\left[e^{\lambda E_{t}}\right]<\infty$ for all $t>0$ and $\lambda>0$ (see [14, 22]).

Proposition 2. Let $E$ be the inverse of a subordinator $D$ with Laplace exponent $\psi$ and infinite Lévy measure $\nu$ in (5). Let $g(s)$ and $R(s)$ be defined as in (6). Fix $\lambda>0, t>0$ and $r>0$.

(1) If there exist a constant $\varepsilon>0$ and a function $x(s):[M, \infty) \rightarrow(g(\infty), g(0))$ with $M>0$ such that $s x(s)>t$ and $R\left(g^{-1}(x(s))\right) / s^{r-1} \geq \lambda+\varepsilon$ for all $s \geq M$, then $\mathbb{E}\left[e^{\lambda E_{t}^{r}}\right]<\infty$.

(2) If there exist a constant $\varepsilon>0$ and a decreasing function $x(s):[M, \infty) \rightarrow(g(\infty), g(0))$ with $M>0$ such that $s x(s)<t$ and $R\left(g^{-1}(x(s))\right) / s^{r-1} \leq \lambda-\varepsilon$ for all $s \geq M$, then $\mathbb{E}\left[e^{\lambda E_{t}^{r}}\right]=\infty$.

Proof. (1) By the assumption and Lemma 5.2(i) of [13],

$$
\mathbb{P}\left(D_{s}<t\right) \leq \mathbb{P}\left(D_{s} \leq s x(s)\right) \leq e^{-s R\left(g^{-1}(x(s))\right)} \leq e^{-(\lambda+\varepsilon) s^{r}}
$$

for all $s \geq M$. Note that

$$
\mathbb{E}\left[e^{\lambda E_{t}^{r}}\right]=\mathbb{E}\left[\left(e^{E_{t}^{r}}\right)^{\lambda}\right]=\int_{0}^{e^{M^{r}}} \lambda z^{\lambda-1} \mathbb{P}\left(e^{E_{t}^{r}}>z\right) \mathrm{d} z+\int_{e^{M^{r}}}^{\infty} \lambda z^{\lambda-1} \mathbb{P}\left(e^{E_{t}^{r}}>z\right) \mathrm{d} z .
$$

Since the first integral on the right hand side is finite, whether the expectation exists or not is completely determined by the second integral, which is estimated with the help of (7) and the change of variables $z=e^{s^{r}}$ as

$$
\int_{e^{M^{r}}}^{\infty} \lambda z^{\lambda-1} \mathbb{P}\left(e^{E_{t}^{r}}>z\right) \mathrm{d} z=\int_{M}^{\infty} \lambda r s^{r-1} e^{\lambda s^{r}} \mathbb{P}\left(D_{s}<t\right) \mathrm{d} s \leq \int_{M}^{\infty} \lambda r s^{r-1} e^{-\varepsilon s^{r}} \mathrm{~d} s .
$$

Since the latter integral is finite, it follows that $\mathbb{E}\left[e^{\lambda E_{t}^{r}}\right]<\infty$.

(2) By the assumption and Lemma 5.2(ii) of [13], there exists a constant $c>0$ such that for all $\eta>0$ and $s \geq M$

$$
\mathbb{P}\left(D_{s}<t\right) \geq \mathbb{P}\left(D_{s} \leq s x(s)\right) \geq\left(1-\frac{(1+\eta) c}{\eta^{2} s R\left(g^{-1}(x(s))\right)}\right) e^{-(1+2 \eta) s R\left(g^{-1}(x(s))\right)} .
$$

With the choice of $\eta=\varepsilon^{2} /\left(2\left(\lambda^{2}-\varepsilon^{2}\right)\right)$, we can find a constant $M_{1} \geq M$ with

$$
\frac{(1+\eta) c}{\eta^{2} s R\left(g^{-1}\left(x\left(M_{1}\right)\right)\right)} \leq \frac{1}{2}
$$

for all $s \geq M_{1}$ (since the fraction on the left hand side goes to 0 as $s \rightarrow \infty$ ). The assumption that $x(s)$ is decreasing together with the fact that $g(s)$ is also decreasing implies $g^{-1}(x(s))$ and $R\left(g^{-1}(x(s))\right)$ are both increasing. Consequently, by the identity $1+2 \eta=\lambda^{2} /\left(\lambda^{2}-\varepsilon^{2}\right)$ and the above estimates, it follows that $\mathbb{P}\left(D_{s}<t\right) \geq(1 / 2) e^{-\lambda^{2} s^{r} /(\lambda+\varepsilon)}$ for $s \geq M_{1}$. This, along with the change of variables $z=e^{s^{r}}$, gives a lower bound for $\int_{e^{M}}^{\infty} \lambda z^{\lambda-1} \mathbb{P}\left(e^{E_{t}^{r}}>z\right) \mathrm{d} z$ as

$$
\int_{M_{1}}^{\infty} \lambda r s^{r-1} e^{\lambda s^{r}} \mathbb{P}\left(D_{s}<t\right) \mathrm{d} s \geq \frac{\lambda}{2} r M_{1}^{r-1} \int_{M_{1}}^{\infty} e^{\lambda \varepsilon s^{r} /(\lambda+\varepsilon)} \mathrm{d} s=\infty
$$

This implies that $\mathbb{E}\left[e^{\lambda E_{t}^{r}}\right]=\infty$.

Corollary 3. Let $E$ be the inverse of a subordinator $D$ with Laplace exponent $\psi$ and infinite Lévy measure $\nu$ in (5). Let $g(s)$ and $R(s)$ be defined as in (6). Fix $\lambda>0, t>0$ and $r>0$.

(1) If there exists a function $x(s)$ defined for large $s$ and taking values in the interval $(g(\infty), g(0))$ such that $s x(s) \rightarrow \infty$ and $R\left(g^{-1}(x(s))\right) / s^{r-1} \rightarrow \infty$ as $s \rightarrow \infty$, then $\mathbb{E}\left[e^{\lambda E_{t}^{r}}\right]<\infty$.

(2) If there exists a decreasing function $x(s)$ defined for large $s$ and taking values in the interval $(g(\infty), g(0))$ such that $s x(s) \rightarrow 0$ and $R\left(g^{-1}(x(s))\right) / s^{r-1} \rightarrow 0$ as $s \rightarrow \infty$, then $\mathbb{E}\left[e^{\lambda E_{t}^{r}}\right]=\infty$. 
We now apply Corollary 3 and the following lemma (in Propositions 1.5.1 and 1.5.7 of [2]) to prove Theorem 1.

Lemma 4. (1) Given $f \in \mathrm{RV}_{\alpha}, f(\infty)=\infty$ if $\alpha>0$, and $f(\infty)=0$ if $\alpha<0$.

(2) If $f_{i} \in \mathrm{RV}_{\alpha_{i}}$ for $i=1,2$ and $f_{2}(\infty)=\infty$, then $f_{1} \circ f_{2} \in \mathrm{RV}_{\alpha_{1} \alpha_{2}}$.

(3) If $f_{i} \in \mathrm{RV}_{\alpha_{i}}$ for $i=1,2$, then $f_{1} \cdot f_{2} \in \mathrm{RV}_{\alpha_{1}+\alpha_{2}}$.

Proof of Theorem 1. By Lemma 4(1), $\psi(\infty)=\infty$ if $\beta \in(0,1)$. On the other hand, if $\beta=0$, by assumption, $\psi(\infty)=\nu(0, \infty)=\infty$. In any case, for any $c>0$, L'Hospital's rule gives

$$
c^{\beta}=\lim _{s \rightarrow \infty} \frac{\psi(c s)}{\psi(s)}=c \lim _{s \rightarrow \infty} \frac{\psi^{\prime}(c s)}{\psi^{\prime}(s)}, \text { or } \lim _{s \rightarrow \infty} \frac{\psi^{\prime}(c s)}{\psi^{\prime}(s)}=c^{\beta-1},
$$

so $\psi^{\prime} \in \mathrm{RV}_{\beta-1}$. Moreover, since $\beta-1<0$, we have $\psi^{\prime}(\infty)=0$ due to Lemma 4(1), so it follows from L'Hospital's rule again that for any $c>0, \lim _{s \rightarrow \infty} \psi^{\prime \prime}(c s) / \psi^{\prime \prime}(s)=c^{\beta-2}$. Consequently, $-\psi^{\prime \prime} \in \mathrm{RV}_{\beta-2}$. (Note that $\psi^{\prime \prime}<0$ as $\psi$ is a Bernstein function.)

Letting $x(s):=g\left(s^{r}\right)=\psi^{\prime}\left(s^{r}\right)$ and using Lemma 4(2)(3) yields

$$
s x(s) \in \mathrm{RV}_{(\beta-1) r+1},
$$

while $R^{\prime}(s)=\psi^{\prime}(s)-\left(s \psi^{\prime}(s)\right)^{\prime}=s\left(-\psi^{\prime \prime}(s)\right) \in \mathrm{RV}_{\beta-1}$. Write $R^{\prime}(s)$ as $s^{\beta-1} \ell(s)$ with a slowly varying function $\ell(s)$. If $\beta \in(0,1)$, then using Karamata's integral theorem (see Proposition 1.5.8 of [2]) yields

$$
R(s)=\int_{0}^{s} R^{\prime}(r) \mathrm{d} r=\int_{0}^{s} r^{\beta-1} \ell(r) \mathrm{d} r \sim \ell(s) \int_{0}^{s} r^{\beta-1} \mathrm{~d} r=\frac{s^{\beta} \ell(s)}{\beta}
$$

as $s \rightarrow \infty$, so $R \in \mathrm{RV}_{\beta}$. On the other hand, if $\beta=0$, then $R \in \mathrm{RV}_{0}$ by Proposition 1.5.9a of [2]. Thus, $R \in \mathrm{RV}_{\beta}$ regardless of the value of $\beta$. Hence,

$$
\frac{R\left(g^{-1}(x(s))\right)}{s^{r-1}}=\frac{R\left(s^{r}\right)}{s^{r-1}} \in \mathrm{RV}_{\beta r-(r-1)}=\mathrm{RV}_{(\beta-1) r+1} .
$$

Again, by Lemma 4(1), the two quantities $s x(s)$ and $R\left(g^{-1}(x(s))\right) / s^{r-1}$ both increase to $\infty$ if $(\beta-1) r+$ $1>0$ and both decrease to 0 if $(\beta-1) r+1<0$. Application of Corollary 3 completes the proof.

Example 5. Fix $\lambda>0$ and $t>0$.

(1) If $\psi(s)=(s+\kappa)^{\beta}-\kappa^{\beta}$ with $\beta \in(0,1)$ and $\kappa \geq 0$, then $\psi \in \mathrm{RV}_{\beta}$ regardless of the value of $\kappa$, so $\mathbb{E}\left[e^{\lambda E_{t}^{r}}\right]<\infty$ as long as $r<1 /(1-\beta)$. This particularly implies that if the subordinator $D$ is stable or tempered stable with stability index $\beta \in(1 / 2,1)$, then $\mathbb{E}\left[e^{\lambda E_{t}^{2}}\right]<\infty$. This fact will be used in the proof of Theorem 7 .

(2) If $\psi \in \mathrm{RV}_{0}$ and $\psi(\infty)=\infty$ (e.g. $\psi(s)=\log (1+s)$, which corresponds to a Gamma subordinator $D)$, then $\mathbb{E}\left[e^{\lambda E_{t}^{r}}\right]<\infty$ for any $0<r \leq 1$, and $\mathbb{E}\left[e^{\lambda E_{t}^{r}}\right]=\infty$ for any $r>1$.

(3) The subordinator $D$ with Laplace exponent $\psi(s)=\int_{0}^{1} s^{\beta} \rho(\mathrm{d} \beta)$, where $\rho$ is a finite Borel measure on $(0,1)$ with $\operatorname{supp}(\rho) \subset(0,1)$, is regarded as a mixture of independent stable subordinators. Its inverse $E$ can be used as a time change introducing more than one subdiffusive mode. In particular, Theorems 3.5 and 3.6 of [9] establish that a class of SDEs driven by a time-changed Lévy process with this particular time change is associated with a class of time-distributed fractional-order pseudo-differential equations. For specific applications where several subdiffusive modes appear, see e.g. [5]. If $\rho=\sum_{j=1}^{J} a_{j} \delta_{\beta_{j}}$, where for each $j, a_{j}>0$ and $\delta_{\beta_{j}}$ is a Dirac measure with mass at $\beta_{j} \in(0,1)$, then since $\psi \in \mathrm{RV}_{\hat{\beta}}$ with $\hat{\beta}:=\max _{1 \leq j \leq J} \beta_{j}$, it follows from Theorem 1 that $\mathbb{E}\left[e^{\lambda E_{t}^{r}}\right]<\infty$ for $0<r<1 /(1-\hat{\beta})$, and $\mathbb{E}\left[e^{\lambda E_{t}^{r}}\right]=\infty$ for $r>1 /(1-\hat{\beta})$.

Remark 6. (1) Corollary 3 can be possibly applied to subordinators whose Laplace exponents are regularly varying at $\infty$ with index 1 . For example, suppose $\widehat{\psi}(s):=a s+\psi(s)$, where $a>0$ and $\psi \in \mathrm{RV}_{\beta}$ with $\beta \in[0,1)$ as in Theorem 1. Then $\widehat{\psi} \in \mathrm{RV}_{1}, \widehat{\psi}^{\prime}=a+\psi^{\prime} \in \mathrm{RV}_{0}$, and $-\widehat{\psi}^{\prime \prime}=-\psi^{\prime \prime} \in \mathrm{RV}_{\beta-2}$. Consequently, letting $x(s):=g\left(s^{r}\right)$ yields $s x(s) \in \mathrm{RV}_{1}$ and $R\left(g^{-1}(x(s))\right) / s^{r-1} \in \mathrm{RV}_{(\beta-1) r+1}$, so both 
quantities go to $\infty$ as $s \rightarrow \infty$ if $(\beta-1) r+1>0$. Thus, application of Corollary 3 yields $\mathbb{E}\left[e^{\lambda E_{t}^{r}}\right]<\infty$ for $r<1 /(1-\beta)$. This implies that Theorem 7 is applicable to subordinators with such Laplace exponents $\widehat{\psi} \in \mathrm{RV}_{1}$.

(2) If $D$ is stable with index $\beta \in(0,1)$, Theorem 1 can be obtained immediately from the known result about the moments of $E_{t}$ (see e.g. Corollary 3.1 of [25] and Proposition 5.6 of [37]) together with the ratio test. Indeed,

$$
\mathbb{E}\left[e^{\lambda E_{t}^{r}}\right]=\sum_{n=0}^{\infty} \frac{\lambda^{n} \mathbb{E}\left[E_{t}^{r n}\right]}{n !}=\sum_{n=0}^{\infty} \frac{\lambda^{n}}{n !} \frac{\Gamma(r n+1)}{\Gamma(r n \beta+1)} t^{r n \beta}=f\left(\lambda t^{r \beta}\right),
$$

where $f(z):=\sum_{n=0}^{\infty} a_{n} z^{n}$ with $a_{n}:=\Gamma(r n+1) /(n ! \Gamma(r n \beta+1))$. By Stirling's formula, as $n \rightarrow \infty$,

$$
\begin{aligned}
\frac{a_{n+1}}{a_{n}} & =\frac{1}{n+1} \cdot \frac{\Gamma(r n+r+1)}{\Gamma(r n+1)} \cdot \frac{\Gamma(r n \beta+1)}{\Gamma(r(n+1) \beta+1)} \\
& \sim \frac{1}{n+1} \cdot \frac{(r n+r)^{r n+r+1 / 2} e^{-(r n+r)}}{(r n)^{r n+1 / 2} e^{-r n}} \cdot \frac{(r n \beta)^{r n \beta+1 / 2} e^{-r n \beta}}{(r(n+1) \beta)^{r(n+1) \beta+1 / 2} e^{-r(n+1) \beta}} \\
& =\frac{1}{n+1} \cdot\left(\frac{n+1}{n}\right)^{r n+1 / 2} \cdot \frac{(r(n+1))^{r}}{e^{r}} \cdot\left(\frac{n}{n+1}\right)^{r n \beta+1 / 2} \cdot \frac{e^{r \beta}}{(r(n+1) \beta)^{r \beta}} \\
& \sim \frac{1}{n+1} \cdot \frac{(r(n+1))^{r}}{(r(n+1) \beta)^{r \beta}} \\
& \longrightarrow \begin{cases}0 & \text { if } r<r \beta+1, \\
\infty & \text { if } r>r \beta+1, \\
r^{r} /(r-1)^{r-1} & \text { if } r=r \beta+1 .\end{cases}
\end{aligned}
$$

This yields Theorem 1. It also follows that in the threshold case when $r=1 /(1-\beta), \mathbb{E}\left[e^{\lambda E_{t}^{r}}\right]<\infty$ if $\lambda t^{r-1}<(r-1)^{r-1} / r^{r}$, while $\mathbb{E}\left[e^{\lambda E_{t}^{r}}\right]=\infty$ if $\lambda t^{r-1}>(r-1)^{r-1} / r^{r}$. This can also be verified using Proposition 2.

\section{Approximation of SDEs with space-time-dependent coeffi- cients}

Suppose the probability space $(\Omega, \mathcal{F}, \mathbb{P})$ is equipped with a filtration $\left(\mathcal{F}_{t}\right)_{t \geq 0}$ satisfying the usual conditions. Let $B$ be an $m$-dimensional $\left(\mathcal{F}_{t}\right)$-adapted Brownian motion which is independent of an $\left(\mathcal{F}_{t}\right)$ adapted subordinator $D$ with infinite Lévy measure. Let $E$ be the inverse of $D$. Consider the SDE

$$
X_{t}=x_{0}+\int_{0}^{t} F\left(s, X_{s}\right) \mathrm{d} E_{s}+\int_{0}^{t} G\left(s, X_{s}\right) \mathrm{d} B_{E_{s}} \text { for } t \in[0, T]
$$

where $x_{0} \in \mathbb{R}^{d}$ is a non-random constant, $T>0$ is a fixed time horizon, and $F(t, x):[0, T] \times \mathbb{R}^{d} \rightarrow \mathbb{R}^{d}$ and $G(t, x):[0, T] \times \mathbb{R}^{d} \rightarrow \mathbb{R}^{d \times m}$ are measurable functions for which there exist constants $K>0$, $\theta_{F} \in(0,1]$ and $\theta_{G} \in(0,1]$ such that

$$
\begin{aligned}
& |F(t, x)-F(t, y)|+|G(t, x)-G(t, y)| \leq K|x-y|, \\
& |F(t, x)|+|G(t, x)| \leq K(1+|x|) \\
& |F(s, x)-F(t, x)| \leq K(1+|x|)|s-t|^{\theta_{F}} \\
& |G(s, x)-G(t, x)| \leq K(1+|x|)|s-t|^{\theta_{G}}
\end{aligned}
$$

for all $x, y \in \mathbb{R}^{d}$ and $s, t \in[0, T]$, with $|\cdot|$ denoting the Euclidean norms of appropriate dimensions. In the remainder of the paper, we assume that $m=d=1$ for simplicity of discussions and expressions; an extension to a multidimensional case is straightforward. For each fixed $t \geq 0$ the random time $E_{t}$ is an $\left(\mathcal{F}_{t}\right)$-stopping time, and therefore, the time-changed filtration $\left(\mathcal{F}_{E_{t}}\right)_{t \geq 0}$ is well-defined. Moreover, since 
the time change $E$ is an $\left(\mathcal{F}_{E_{t}}\right)$-adapted nondecreasing process and the time-changed Brownian motion $B \circ E=\left(B_{E_{t}}\right)_{t \geq 0}$ is an $\left(\mathcal{F}_{E_{t}}\right)$-martingale, $\operatorname{SDE}(8)$ is understood within the framework of stochastic integrals driven by semimartingales (see Corollary 10.12 of [11]; also see [17] for details). Conditions (9)(10) guarantee the existence of a unique strong solution of SDE (8) which is $\left(\mathcal{F}_{E_{t}}\right)$-adapted. Conditions (11)-(12) are required to obtain strong convergence of our approximation scheme in Theorem 7 . Note that in the classical setting of an Itô SDE (i.e. SDE (8) with $E_{t} \equiv t$ ), the corresponding theorem for strong approximation usually assumes (11)-(12) with $\theta_{F}=\theta_{G}=1 / 2$ (see Theorem 10.2.2 of [16]). Note also that we exclude cases when $\theta_{F}>1$ and/or $\theta_{G}>1$ since that would imply $F$ and/or $G$ must be independent of $t$ (i.e. $F(t, x)=F(x)$ and $G(t, x)=G(x)$ ).

As noted in Section 1, a standard conditioning approach used in [14] based on the duality principle in [17] no longer works for SDE (8). Our argument in this paper is different. We do not rely on the duality principle. Instead, we utilize a Gronwall-type inequality involving a stochastic driver to control the moment of the error process. Moreover, Theorem 1 established in Section 2 will be used to guarantee that the error bound to be ultimately derived in the proof of Theorem 7 is meaningful.

Fix an equidistant step size $\delta \in(0,1)$ and a time horizon $T>0$. To approximate an inverse subordinator $E$ on the interval $[0, T]$, we follow the idea presented in $[19,20]$. Namely, we first simulate a sample path of the subordinator $D$, which has independent and stationary increments, by setting $D_{0}=0$ and then following the rule $D_{i \delta}:=D_{(i-1) \delta}+Z_{i}, i=1,2,3, \ldots$, with an i.i.d. sequence $\left\{Z_{i}\right\}_{i \in \mathbb{N}}$ distributed as $Z_{i}={ }^{\mathrm{d}} D_{\delta}$. We stop this procedure upon finding the integer $N$ satisfying $T \in\left[D_{N \delta}, D_{(N+1) \delta}\right)$. Note that the $\mathbb{N} \cup\{0\}$-valued random variable $N$ indeed exists since $D_{t} \rightarrow \infty$ as $t \rightarrow \infty$ a.s. To generate the random variables $\left\{Z_{i}\right\}$, one can use algorithms presented in Chapter 6 of [3]. Next, let

$$
E_{t}^{\delta}:=\left(\min \left\{n \in \mathbb{N} ; D_{n \delta}>t\right\}-1\right) \delta, \quad t \in[0, T] .
$$

The sample paths of $E^{\delta}=\left(E_{t}^{\delta}\right)_{t \geq 0}$ are nondecreasing step functions with constant jump size $\delta$ and the $i$ th waiting time given by $Z_{i}=D_{i \delta}-D_{(i-1) \delta}$. Indeed, it is easy to see that for $n=0,1,2, \ldots, N$,

$$
E_{t}^{\delta}=n \delta \quad \text { whenever } t \in\left[D_{n \delta}, D_{(n+1) \delta}\right) .
$$

In particular, $E_{T}^{\delta}=N \delta$. The process $E^{\delta}$ efficiently approximates $E$; indeed, a.s.,

$$
E_{t}-\delta \leq E_{t}^{\delta} \leq E_{t} \text { for all } t \in[0, T]
$$

For proofs, see [14, 20].

Now, let

$$
\tau_{n}=D_{n \delta} \text { for } n=0,1,2, \ldots, N
$$

and let

$$
n_{t}=\max \left\{n \in \mathbb{N} \cup\{0\} ; \tau_{n} \leq t\right\} \text { for } t \geq 0 .
$$

By the independence assumption between $B$ and $D$, we can approximate the Brownian motion $B$ over the time steps $\{0, \delta, 2 \delta, \ldots, N \delta\}$, independently of $D$. Define a discrete-time process $\left(X_{\tau_{n}}^{\delta}\right)_{n \in\{0,1,2, \ldots, N\}}$ by setting

$$
\begin{aligned}
& X_{0}^{\delta}=x_{0}, \\
& X_{\tau_{n+1}}^{\delta}=X_{\tau_{n}}^{\delta}+F\left(\tau_{n}, X_{\tau_{n}}^{\delta}\right) \delta+G\left(\tau_{n}, X_{\tau_{n}}^{\delta}\right)\left(B_{(n+1) \delta}-B_{n \delta}\right)
\end{aligned}
$$

for $n=0,1,2, \ldots, N-1$. Define a continuous-time process $X^{\delta}=\left(X_{t}^{\delta}\right)_{t \in[0, T]}$ by piecewise constant interpolation

$$
X_{t}^{\delta}=X_{\tau_{n_{t}}}^{\delta}
$$

Note that any time point $t \geq 0$ satisfies

$$
\tau_{n_{t}} \leq t<\tau_{n_{t}+1}
$$

and that sample paths of $X^{\delta}$ and $E^{\delta}$ are both constant over any interval of the form $\left[\tau_{n}, \tau_{n+1}\right)$. Figure 2 presents a simulation of sample paths of $E$ and $X$ based on this approximation scheme, where the time component of the external force term is taken to be sinusoidal as in e.g. [36]. 


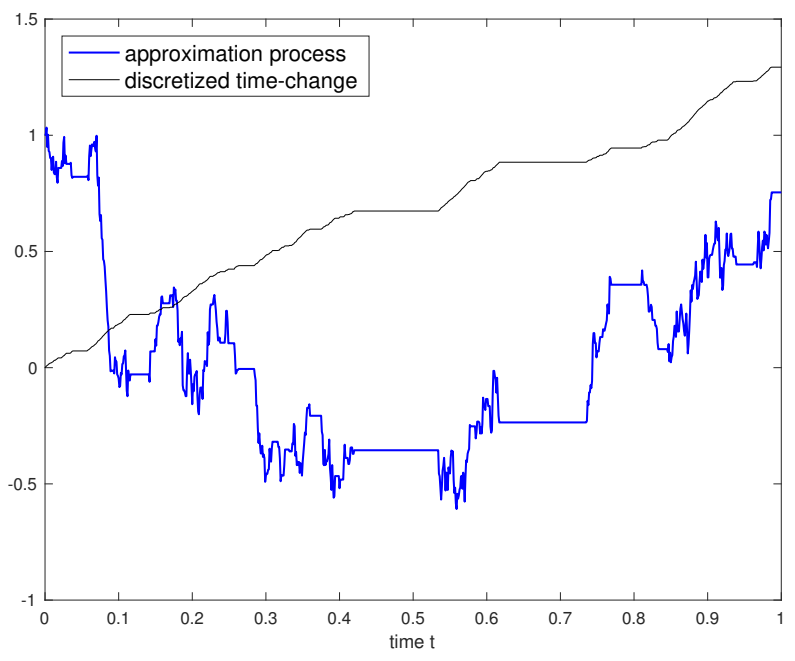

Figure 2: Sample paths of an inverse 0.85-stable subordinator $E$ (black) and the corresponding solution $X$ (blue) of SDE $X_{t}=1+\int_{0}^{t}(\sin s) X_{s} \mathrm{~d} E_{s}+B_{E_{t}}$ on the time interval $[0,1]$.

We now state the main theorem of this paper, which gives the rate of strong convergence of the approximation scheme for SDE (8). Recall that an approximation process $X^{\delta}$ with step size $\delta>0$ is said to converge strongly to the solution $X$ uniformly on $[0, T]$ with order $\eta \in(0, \infty)$ if there exist finite positive constants $C$ and $\delta_{0}$ such that $\mathbb{E}\left[\sup _{0 \leq t \leq T}\left|X_{t}-X_{t}^{\delta}\right|\right] \leq C \delta^{\eta}$ for all $\delta \in\left(0, \delta_{0}\right)$.

Theorem 7. Let $E$ be the inverse of a subordinator $D$ with Laplace exponent $\psi$ and infinite Lévy measure. Let $B$ be Brownian motion independent of $D$. Let $X^{\delta}$ be the approximation process defined in (16)-(18) for the exact solution $X$ of SDE (8), where the coefficients $F(t, x)$ and $G(t, x)$ satisfy conditions (9)-(12). Suppose further that at least one of the following conditions holds:

(i) $\psi$ is regularly varying at $\infty$ with index $\beta \in(1 / 2,1)$;

(ii) $G(t, x)=G(t)$ for all $(t, x) \in[0, T] \times \mathbb{R}$.

Then for any $\varepsilon \in(0,1 / 2)$, there exist constants $C \in(0, \infty)$ (not depending on $\delta$ ) and $\delta_{0}=\delta_{0}(\varepsilon) \in(0,1)$ such that

$$
\mathbb{E}\left[\sup _{0 \leq t \leq T}\left|X_{t}-X_{t}^{\delta}\right|\right] \leq C\left(\delta^{\theta_{F}}+\delta^{\theta_{G}}+\delta^{1 / 2-\varepsilon}\right)
$$

for all $\delta \in\left(0, \delta_{0}\right)$. Thus, $X^{\delta}$ converges strongly to $X$ uniformly on $[0, T]$ with order $\min \left(\theta_{F}, \theta_{G}, 1 / 2-\varepsilon\right)$.

The proof of Theorem 7 is based on the following lemma.

Lemma 8. Let $E$ be the inverse of a subordinator $D$ with infinite Lévy measure. Let $B$ be Brownian motion independent of $D$. Let $X$ be the solution of $S D E$ (8), where the coefficients $F(t, x)$ and $G(t, x)$ satisfy conditions (9)-(10). For a fixed $p \in[1, \infty)$, let $Y_{T}^{(p)}:=1+\sup _{0 \leq s \leq T}\left|X_{s}\right|^{p}$. Then $\mathbb{E}\left[Y_{T}^{(p)}\right]<\infty$.

To prove the lemma, let us recall two inequalities. First, for any $p \in[1, \infty)$, the inequality

$$
(x+y+z)^{p} \leq c_{p}\left(x^{p}+y^{p}+z^{p}\right)
$$

is valid for all $x, y, z \geq 0$, where $c_{p}=3^{p-1}$. Second, the Burkholder-Davis-Gundy inequality states that for any $p>0$, there exists a constant $b_{p}>0$ such that

$$
\mathbb{E}\left[\sup _{0 \leq t \leq S}\left|M_{t}\right|^{p}\right] \leq b_{p} \mathbb{E}\left[[M, M]_{S}^{p / 2}\right]
$$


for any stopping time $S$ and any continuous local martingale $M$ with quadratic variation $[M, M]$. The constant $b_{p}$ can be taken independently of $S$ and $M$; see Proposition 3.26 and Theorem 3.28 of Chapter 3 of $[15]$.

Since the Brownian motion $B$ and the subordinator $D$ are assumed independent, it is possible to set up $B$ and $D$ on a product space with product measure $\mathbb{P}=\mathbb{P}_{B} \times \mathbb{P}_{D}$ with obvious notation. We use this set up in the proofs of Lemma 8 and Theorem 7 below. Let $\mathbb{E}_{B}, \mathbb{E}_{D}$ and $\mathbb{E}$ denote the expectations under the probability measures $\mathbb{P}_{B}, \mathbb{P}_{D}$ and $\mathbb{P}$, respectively.

Proof of Lemma 8. It suffices to prove the statement for $p \geq 2$ since the result for $1 \leq p<2$ follows immediately from the result for $p \geq 2$ with Jensen's inequality. Fix $p \geq 2$ and let $Y_{t}^{(p)}:=1+$ $\sup _{0 \leq s \leq t}\left|X_{s}\right|^{p}$ for $t \in[0, T]$. Let $S_{\ell}:=\inf \left\{t \geq 0 ; Y_{t}^{(p)}>\ell\right\}$ for $\ell \in \mathbb{N}$. Since the solution $X$ has continuous paths, $Y_{t}^{(p)}<\infty$, and hence, $S_{\ell} \uparrow \infty$ as $\ell \rightarrow \infty$. The idea of the proof is to first apply a Gronwall-type inequality to the function $t \mapsto \mathbb{E}_{B}\left[Y_{t \wedge S_{\ell}}^{(p)}\right]$ for a fixed $\ell$ and then let $t=T$ and $\ell \rightarrow \infty$ in the obtained inequality to establish a desired bound for $\mathbb{E}_{B}\left[Y_{T}^{(p)}\right]$. Note that we introduced the localizing sequence $\left\{S_{\ell} ; \ell \in \mathbb{N}\right\}$ in order to guarantee that $\int_{0}^{t} \mathbb{E}_{B}\left[Y_{r \wedge S_{\ell}}^{(p)}\right] \mathrm{d} E_{r} \leq \ell E_{t}<\infty$, which enables us to apply the Gronwall-type inequality.

Fix $\ell \in \mathbb{N}$ and $t \in[0, T]$. Since $E$ has continuous paths, by Theorem 10.17 of [11] (also see Lemma 2.4 and Example 2.5 of [17]), $B \circ E$ is a continuous martingale with quadratic variation $[B \circ E, B \circ E]=$ $[B, B] \circ E=E$. By the Itô formula and representation (8),

$$
X_{s}^{p}=x_{0}^{p}+\int_{0}^{s}\left\{p X_{r}^{p-1} F\left(r, X_{r}\right)+\frac{1}{2} p(p-1) X_{r}^{p-2} G^{2}\left(r, X_{r}\right)\right\} \mathrm{d} E_{r}+M_{s},
$$

where

$$
M_{s}=\int_{0}^{s} p X_{r}^{p-1} G\left(r, X_{r}\right) \mathrm{d} B_{E_{r}} .
$$

Due to condition (10), the absolute value of the integrand of the $\mathrm{d} E_{r}$ integral is dominated by

$$
p K\left|X_{r}\right|^{p-1}\left(1+\left|X_{r}\right|\right)+\frac{1}{2} p(p-1) K^{2}\left|X_{r}\right|^{p-2}\left(1+\left|X_{r}\right|\right)^{2} \leq A_{1} Y_{r}^{(p)},
$$

where $A_{1}=p c_{p} K+p(p-1) c_{p} K^{2} / 2$. Thus,

$$
Y_{t \wedge S_{\ell}}^{(p)}=1+\sup _{0 \leq s \leq t \wedge S_{\ell}}\left|X_{s}\right|^{p} \leq 1+\left|x_{0}\right|^{p}+I_{1}+I_{2},
$$

where

$$
I_{1}=A_{1} \int_{0}^{t \wedge S_{\ell}} Y_{r}^{(p)} \mathrm{d} E_{r} \quad \text { and } \quad I_{2}=\sup _{0 \leq s \leq t \wedge S_{\ell}}\left|M_{s}\right| .
$$

Note that for any nonnegative process $u(r)$, the inequality

$$
\int_{0}^{t \wedge S_{\ell}} u(r) \mathrm{d} E_{r} \leq \int_{0}^{t} u\left(r \wedge S_{\ell}\right) \mathrm{d} E_{r}
$$

holds. Indeed, the inequality obviously holds if $t \leq S_{\ell}$, while if $t>S_{\ell}$, then $\int_{0}^{t} u\left(r \wedge S_{\ell}\right) \mathrm{d} E_{r}=$ $\int_{0}^{S_{\ell}} u(r) \mathrm{d} E_{r}+\int_{S_{\ell}}^{t} u\left(S_{\ell}\right) \mathrm{d} E_{r} \geq \int_{0}^{t \wedge S_{\ell}} u(r) \mathrm{d} E_{r}$, thereby yielding (22). Thus,

$$
\mathbb{E}_{B}\left[I_{1}\right] \leq A_{1} \int_{0}^{t} \mathbb{E}_{B}\left[Y_{r \wedge S_{\ell}}^{(p)}\right] \mathrm{d} E_{r}
$$

To deal with $I_{2}$, note that the stochastic integral $\left(M_{t}\right)_{t \geq 0}$ is a local martingale with quadratic variation $[M, M]_{t}=\int_{0}^{t} p^{2} X_{r}^{2 p-2} G^{2}\left(r, X_{r}\right) \mathrm{d} E_{r}$ since stochastic integration preserves the local martingale property; see Chapter III, Theorem 29 in [33]. By condition (10), for $0 \leq r \leq t \wedge S_{\ell}$,

$$
p^{2} X_{r}^{2 p-2} G^{2}\left(r, X_{r}\right) \leq p^{2} K^{2} X_{r}^{2 p-2}\left(1+\left|X_{r}\right|\right)^{2} \leq p^{2} c_{p}^{2} K^{2} Y_{t \wedge S_{\ell}}^{(p)} Y_{r}^{(p)}
$$


and hence, $\left([M, M]_{t \wedge S_{\ell}}\right)^{1 / 2}$ is dominated by

$$
p c_{p} K\left(Y_{t \wedge S_{\ell}}^{(p)} \int_{0}^{t \wedge S_{\ell}} Y_{r}^{(p)} \mathrm{d} E_{r}\right)^{1 / 2} \leq p c_{p} K\left(\frac{Y_{t \wedge S_{\ell}}^{(p)}}{2 b_{1} p c_{p} K}+2 b_{1} p c_{p} K \int_{0}^{t \wedge S_{\ell}} Y_{r}^{(p)} \mathrm{d} E_{r}\right),
$$

where we used the inequality $(a b)^{1 / 2} \leq a / \lambda+\lambda b$ valid for any $a, b \geq 0$ and $\lambda>0$, and $b_{1}$ is the constant appearing in the Burkholder-Davis-Gundy inequality (20). Applying the latter inequality and inequality (22) now gives

$$
\mathbb{E}_{B}\left[I_{2}\right] \leq b_{1} \mathbb{E}_{B}\left[\left([M, M]_{t \wedge S_{\ell}}\right)^{1 / 2}\right] \leq \frac{1}{2} \mathbb{E}_{B}\left[Y_{t \wedge S_{\ell}}^{(p)}\right]+A_{2} \int_{0}^{t} \mathbb{E}_{B}\left[Y_{r \wedge S_{\ell}}^{(p)}\right] \mathrm{d} E_{r},
$$

where $A_{2}=2 b_{1} p^{2} c_{p}^{2} K^{2}$. Note that the constant $b_{1}$ is independent of the stopping time $t \wedge S_{\ell}$, and in particular, $A_{2}$ does not depend on $\ell$. Taking $\mathbb{E}_{B}$ on both sides of (21) and using (23) and (24) yields

$$
\mathbb{E}_{B}\left[Y_{t \wedge S_{\ell}}^{(p)}\right] \leq 1+\left|x_{0}\right|^{p}+\frac{1}{2} \mathbb{E}_{B}\left[Y_{t \wedge S_{\ell}}^{(p)}\right]+\left(A_{1}+A_{2}\right) \int_{0}^{t} \mathbb{E}_{B}\left[Y_{r \wedge S_{\ell}}^{(p)}\right] \mathrm{d} E_{r},
$$

which in turn gives $\mathbb{E}_{B}\left[Y_{t \wedge S_{\ell}}^{(p)}\right] \leq 2\left(1+\left|x_{0}\right|^{p}\right)+2\left(A_{1}+A_{2}\right) \int_{0}^{t} \mathbb{E}_{B}\left[Y_{r \wedge S_{\ell}}^{(p)}\right] \mathrm{d} E_{r}$. Thus, applying a Gronwalltype inequality in Chapter IX.6a, Lemma 6.3 of [12] yields

$$
\mathbb{E}_{B}\left[Y_{t \wedge S_{\ell}}^{(p)}\right] \leq 2\left(1+\left|x_{0}\right|^{p}\right) e^{2\left(A_{1}+A_{2}\right) E_{t}}
$$

for all $t \in[0, T]$. Note that $E_{t}$ appears in the exponent on the right hand side since the integral above is driven by the process $E$. Setting $t=T$, letting $\ell \rightarrow \infty$ while recalling $A_{1}$ and $A_{2}$ do not depend on $\ell$, and using the monotone convergence theorem yields $\mathbb{E}_{B}\left[Y_{T}^{(p)}\right] \leq 2\left(1+\left|x_{0}\right|^{p}\right) e^{2\left(A_{1}+A_{2}\right) E_{T}}$. Taking $\mathbb{E}_{D}$ on both sides, noting $\mathbb{E}_{D}\left[\mathbb{E}_{B}\left[Y_{T}^{(p)}\right]\right]=\mathbb{E}\left[Y_{T}^{(p)}\right]$, and using the fact that $\mathbb{E}\left[e^{\lambda E_{T}}\right]<\infty$ for any $\lambda>0$ yields the desired result.

Proof of Theorem \%. Let

$$
Z_{t}:=\sup _{0 \leq s \leq t}\left|X_{s}-X_{s}^{\delta}\right| \text { for } t \in[0, T] .
$$

As in the proof of Lemma 8 , we use the localizing sequence $S_{\ell}=\inf \left\{t \geq 0 ; Z_{t+1}>\ell\right\}$, which allows us to safely apply a Gronwall-type inequality. Here, $S_{\ell}$ is defined as $\inf \left\{t \geq 0 ; Z_{t+1}>\ell\right\}$ instead of $\inf \left\{t \geq 0 ; Z_{t}>\ell\right\}$ in order to guarantee that $Z_{t \wedge S_{\ell}} \leq \ell$ even if the process $Z$ exceeds the level $\ell$ by a jump. Note that the approximation $X^{\delta}$ is a piecewise constant process with finitely many jumps, so $Z_{t}<\infty$, and hence, $S_{\ell} \uparrow \infty$ as $\ell \rightarrow \infty$. In the remainder of the proof, however, to clarify the main ideas, we assume the function $t \mapsto Z_{t}$ is bounded.

By (16)-(18),

$$
X_{s}^{\delta}-x_{0}=\sum_{i=0}^{n_{s}-1}\left(X_{\tau_{i+1}}^{\delta}-X_{\tau_{i}}^{\delta}\right)=\sum_{i=0}^{n_{s}-1}\left(F\left(\tau_{i}, X_{\tau_{i}}^{\delta}\right) \delta+G\left(\tau_{i}, X_{\tau_{i}}^{\delta}\right)\left(B_{(i+1) \delta}-B_{i \delta}\right)\right) .
$$

Note that $E_{\tau_{i}}=E_{D_{i \delta}}=i \delta$ and that $\tau_{i}=\tau_{n_{r}}$ for any $r \in\left[\tau_{i}, \tau_{i+1}\right)$, which implies the above can be rewritten as

$$
X_{s}^{\delta}-x_{0}=\int_{0}^{\tau_{n_{s}}} F\left(\tau_{n_{r}}, X_{r}^{\delta}\right) \mathrm{d} E_{r}+\int_{0}^{\tau_{n_{s}}} G\left(\tau_{n_{r}}, X_{r}^{\delta}\right) \mathrm{d} B_{E_{r}} .
$$

Hence, for a fixed $t \in[0, T]$,

$$
Z_{t} \leq I_{1}+I_{2}+I_{3}+I_{4}, \quad Z_{t}^{2} \leq 4\left(I_{1}^{2}+I_{2}^{2}+I_{3}^{2}+I_{4}^{2}\right),
$$

where

$$
\begin{aligned}
& I_{1}=\sup _{0 \leq s \leq t}\left|\int_{0}^{\tau_{n_{s}}}\left(F\left(r, X_{r}\right)-F\left(\tau_{n_{r}}, X_{r}^{\delta}\right)\right) \mathrm{d} E_{r}\right|, \quad I_{2}=\sup _{0 \leq s \leq t}\left|\int_{\tau_{n_{s}}}^{s} F\left(r, X_{r}\right) \mathrm{d} E_{r}\right|, \\
& I_{3}=\sup _{0 \leq s \leq t}\left|\int_{0}^{\tau_{n_{s}}}\left(G\left(r, X_{r}\right)-G\left(\tau_{n_{r}}, X_{r}^{\delta}\right)\right) \mathrm{d} B_{E_{r}}\right|, I_{4}=\sup _{0 \leq s \leq t}\left|\int_{\tau_{n_{s}}}^{s} G\left(r, X_{r}\right) \mathrm{d} B_{E_{r}}\right| .
\end{aligned}
$$


In terms of $I_{1}$, note that for $r \in\left[0, \tau_{n_{t}}\right)$, by conditions (9) and (11),

$$
\begin{aligned}
\left|F\left(r, X_{r}\right)-F\left(\tau_{n_{r}}, X_{r}^{\delta}\right)\right| & \leq\left|F\left(r, X_{r}\right)-F\left(\tau_{n_{r}}, X_{r}\right)\right|+\left|F\left(\tau_{n_{r}}, X_{r}\right)-F\left(\tau_{n_{r}}, X_{r}^{\delta}\right)\right| \\
& \leq K\left(1+\left|X_{r}\right|\right)\left(r-\tau_{n_{r}}\right)^{\theta_{F}}+K\left|X_{r}-X_{r}^{\delta}\right| \\
& \leq K Y_{\tau_{n_{t}}}^{(1)}\left(\tau_{n_{r}+1}-\tau_{n_{r}}\right)^{\theta_{F}}+K Z_{r},
\end{aligned}
$$

where $Y_{t}^{(p)}=1+\sup _{0 \leq s \leq t}\left|X_{s}\right|^{p}$ as in the proof of Lemma 8. The function $g(x):=x^{1 / \theta_{F}}(x \geq 0)$ is convex since $\theta_{F} \in(0,1]$, so by Jensen's inequality,

$$
\begin{aligned}
& \left(\sum_{i=0}^{n_{t}-1}\left(\tau_{i+1}-\tau_{i}\right)^{\theta_{F}}\right)^{1 / \theta_{F}}=g\left(\frac{1}{n_{t}} \sum_{i=0}^{n_{t}-1} n_{t}\left(\tau_{i+1}-\tau_{i}\right)^{\theta_{F}}\right) \\
& \leq \frac{1}{n_{t}} \sum_{i=0}^{n_{t}-1} g\left(n_{t}\left(\tau_{i+1}-\tau_{i}\right)^{\theta_{F}}\right)=n_{t}^{1 / \theta_{F}-1} \sum_{i=0}^{n_{t}-1}\left(\tau_{i+1}-\tau_{i}\right)=n_{t}^{1 / \theta_{F}-1} \tau_{n_{t}} .
\end{aligned}
$$

This, together with the identity $\int_{\tau_{i}}^{\tau_{i+1}}\left(\tau_{n_{r}+1}-\tau_{n_{r}}\right)^{\theta_{F}} \mathrm{~d} E_{r}=\delta\left(\tau_{i+1}-\tau_{i}\right)^{\theta_{F}}$, yields

$$
\int_{0}^{\tau_{n_{t}}}\left(\tau_{n_{r}+1}-\tau_{n_{r}}\right)^{\theta_{F}} \mathrm{~d} E_{r}=\delta \sum_{i=0}^{n_{t}-1}\left(\tau_{i+1}-\tau_{i}\right)^{\theta_{F}} \leq \delta n_{t}^{1-\theta_{F}} \tau_{n_{t}}^{\theta_{F}}
$$

By (14)-(15), $n_{t}$ is a random variable satisfying the relation $n_{t} \delta=E_{t}^{\delta} \leq E_{t}$. Using the inequalities $\tau_{n_{t}} \leq t \leq T$ and putting together (26) and (27) yields

$$
I_{1} \leq K \delta^{\theta_{F}} T^{\theta_{F}} E_{T}^{1-\theta_{F}} Y_{T}^{(1)}+K \int_{0}^{t} Z_{r} \mathrm{~d} E_{r}
$$

By the Cauchy-Schwarz inequality, this implies

$$
I_{1}^{2} \leq 4 K^{2} \delta^{2 \theta_{F}} T^{2 \theta_{F}} E_{T}^{2\left(1-\theta_{F}\right)} Y_{T}^{(2)}+2 K^{2} E_{T} \int_{0}^{t} Z_{r}^{2} \mathrm{~d} E_{r} .
$$

On the other hand, in terms of $I_{2}$, by condition (10),

$$
I_{2} \leq \sup _{0 \leq s \leq t} K \int_{\tau_{n_{s}}}^{s}\left(1+\left|X_{r}\right|\right) \mathrm{d} E_{r} \leq \sup _{0 \leq s \leq t} K Y_{s}^{(1)}\left(E_{s}-E_{\tau_{n_{s}}}\right) \leq K \delta Y_{T}^{(1)},
$$

which implies

$$
I_{2}^{2} \leq 2 K^{2} \delta^{2} Y_{T}^{(2)}
$$

To deal with $I_{3}$, note that $B \circ E$ is a martingale with quadratic variation $E$, and hence, by the Burkholder-Davis-Gundy inequality (20),

$$
\begin{aligned}
\mathbb{E}_{B}\left[I_{3}^{2}\right] & \leq \mathbb{E}_{B}\left[\sup _{0 \leq s \leq \tau_{n_{t}}}\left|\int_{0}^{s}\left(G\left(r, X_{r}\right)-G\left(\tau_{n_{r}}, X_{r}^{\delta}\right)\right) \mathrm{d} B_{E_{r}}\right|^{2}\right] \\
& \leq b_{2} \mathbb{E}_{B}\left[\int_{0}^{\tau_{n_{t}}}\left(G\left(r, X_{r}\right)-G\left(\tau_{n_{r}}, X_{r}^{\delta}\right)\right)^{2} \mathrm{~d} E_{r}\right] .
\end{aligned}
$$

An estimation similar to the one in (26) gives

$$
\mathbb{E}_{B}\left[I_{3}^{2}\right] \leq 4 K^{2} b_{2} \mathbb{E}_{B}\left[Y_{\tau_{n_{t}}}^{(2)}\right] \int_{0}^{\tau_{n_{t}}}\left(\tau_{n_{r}+1}-\tau_{n_{r}}\right)^{2 \theta_{G}} \mathrm{~d} E_{r}+2 K^{2} b_{2} \int_{0}^{\tau_{n_{t}}} \mathbb{E}_{B}\left[Z_{r}^{2}\right] \mathrm{d} E_{r} .
$$


Note that for $\theta_{G} \in(1 / 2,1]$, we have $|t-s|^{\theta_{G}} \leq(2 T)^{\theta_{G}-1 / 2}|t-s|^{1 / 2}$, so there is no loss of generality in assuming $\theta_{G} \in(0,1 / 2]$ in (12). Assuming $\theta_{G} \in(0,1 / 2]$, we can use (27) with $\theta_{F} \in(0,1]$ replaced by $2 \theta_{G}$, yielding

$$
\begin{aligned}
\mathbb{E}_{B}\left[I_{3}^{2}\right] & \leq 4 K^{2} b_{2} \mathbb{E}_{B}\left[Y_{\tau_{n_{t}}}^{(2)}\right] \delta n_{t}^{1-2 \theta_{G}} \tau_{n_{t}}^{2 \theta_{G}}+2 K^{2} b_{2} \int_{0}^{\tau_{n t}} \mathbb{E}_{B}\left[Z_{r}^{2}\right] \mathrm{d} E_{r} \\
& \leq 4 \delta^{2 \theta_{G}} K^{2} b_{2} T^{2 \theta_{G}} E_{T}^{1-2 \theta_{G}} \mathbb{E}_{B}\left[Y_{T}^{(2)}\right]+2 K^{2} b_{2} \int_{0}^{t} \mathbb{E}_{B}\left[Z_{r}^{2}\right] \mathrm{d} E_{r},
\end{aligned}
$$

where we used $n_{t} \delta=E_{t}^{\delta} \leq E_{t}$ and $\tau_{n_{t}} \leq t \leq T$.

Estimation of $I_{4}^{2}$ requires some careful work. By the change-of-variable formula for stochastic integrals driven by time-changed semimartingales (Theorem 3.1 of [17]) and the relation $E_{\tau_{n_{s}}}=n_{s} \delta=E_{s}^{\delta}$, it follows that

$$
\mathbb{E}_{B}\left[I_{4}^{2}\right]=\mathbb{E}_{B}\left[\sup _{0 \leq s \leq t}\left|\int_{E_{s}^{\delta}}^{E_{s}} G\left(D_{v-}, X_{D_{v-}}\right) \mathrm{d} B_{v}\right|^{2}\right] \leq \mathbb{E}_{B}\left[\sup _{0 \leq r, s \leq E_{T}, 0 \leq s-r \leq \delta} N_{r, s}^{2}\right],
$$

where $N_{r, s}=\int_{r}^{s} G\left(D_{v-}, X_{D_{v_{-}}}\right) \mathrm{d} B_{v}$. Note that the use of the change-of-variable formula requires that the semimartingale ( $B$ in this case) be constant on any interval of the form $\left[E_{t-}, E_{t}\right]$, but this is satisfied since the time change $E$ has continuous paths. Observe that

$$
\begin{aligned}
& \mathbb{E}_{B}\left[I_{4}^{2}\right]=\mathbb{E}_{B}\left[\mathbf{1}_{E_{T} \geq \delta} \cdot I_{4}^{2}\right]+\mathbb{E}_{B}\left[\mathbf{1}_{E_{T}<\delta} \cdot I_{4}^{2}\right] \\
& \leq \mathbb{E}_{B}\left[\mathbf{1}_{E_{T} \geq \delta} \cdot \sup _{0 \leq r, s \leq E_{T}, 0 \leq s-r \leq \delta} N_{r, s}^{2}\right]+\mathbb{E}_{B}\left[\sup _{0 \leq r, s \leq \delta, 0 \leq s-r \leq \delta} N_{r, s}^{2}\right],
\end{aligned}
$$

where $\mathbf{1}_{A}$ denotes the indicator function on a set $A$. Also, for all $0 \leq r<s \leq u$,

$$
\int_{r}^{s} G^{2}\left(D_{v-}, X_{D_{v-}}\right) \mathrm{d} v \leq K^{2} \int_{r}^{s}\left(1+\left|X_{D_{v-}}\right|\right)^{2} \mathrm{~d} v \leq \xi(u)|s-r|,
$$

where $\xi(u):=2 K^{2} Y_{D_{u-}}^{(2)}$. By Lemma 8 , for $\alpha>0$,

$$
\mathbb{E}_{B}\left[\xi\left(E_{T}\right)^{1+\alpha}\right] \leq\left(2 K^{2}\right)^{1+\alpha} c_{1+\alpha} \mathbb{E}_{B}\left[Y_{T}^{(2(1+\alpha))}\right]<\infty
$$

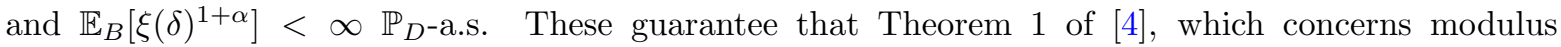
of continuity for stochastic integrals, is applicable. Namely, there exists a constant $C_{1}$ such that $\mathbb{E}_{B}\left[\sup _{0 \leq r, s \leq u, 0 \leq s-r \leq \delta} N_{r, s}^{2}\right] \leq C_{1} \delta \log (2 u / \delta)$ for all $0<\delta \leq u$ with $u=E_{T}$ and $u=\delta$, and their proof shows that $C_{1}$ can be taken independently of $u$. This, together with (33), gives

$$
\mathbb{E}_{B}\left[I_{4}^{2}\right] \leq C_{1} \delta \log \left(\frac{2 E_{T}}{\delta}\right)+C_{1} \delta \log 2=C_{1} \delta \log \left(\frac{4 E_{T}}{\delta}\right) .
$$

Now, let us assume that condition (i) holds; i.e. $\psi \in \mathrm{RV}_{\beta}$ with $\beta \in(1 / 2,1)$. Putting together the estimates (25), (29), (31), (32) and (34) gives

$$
\mathbb{E}_{B}\left[Z_{t}^{2}\right] \leq\left(V_{1}+V_{2}\right)+8 K^{2}\left(E_{T}+b_{2}\right) \int_{0}^{t} \mathbb{E}_{B}\left[Z_{r}^{2}\right] \mathrm{d} E_{r},
$$

where

$$
V_{1}=4 C_{1} \delta \log \left(\frac{4 E_{T}}{\delta}\right), \quad V_{2}=C_{2}\left(\delta^{2}+E_{T}^{2\left(1-\theta_{F}\right)} \delta^{2 \theta_{F}}+E_{T}^{1-2 \theta_{G}} \delta^{2 \theta_{G}}\right) \mathbb{E}_{B}\left[Y_{T}^{(2)}\right]
$$

with $C_{2}$ being a constant depending on $K, T, \theta_{F}$ and $\theta_{G}$. Applying a Gronwall-type inequality in Chapter IX.6a, Lemma 6.3 of [12] and taking $\mathbb{E}_{D}$ on both sides of the obtained inequality with $t=T$ gives

$$
\mathbb{E}\left[Z_{T}^{2}\right] \leq \mathbb{E}\left[\left(V_{1}+V_{2}\right) e^{8 K^{2}\left(E_{T}+b_{2}\right) E_{T}}\right] \leq\left(\mathbb{E}\left[\left(V_{1}+V_{2}\right)^{2}\right] \cdot \mathbb{E}\left[e^{16 K^{2}\left(E_{T}+b_{2}\right) E_{T}}\right]\right)^{1 / 2}
$$


The assumption $\beta \in(1 / 2,1)$ allows us to use Theorem 1 with $r=2<1 /(1-\beta)$, which implies $\mathbb{E}\left[e^{16 K^{2}\left(E_{T}+b_{2}\right) E_{T}}\right]<\infty$. Moreover, $\mathbb{E}\left[V_{2}^{2}\right]<\infty$ since $\mathbb{E}\left[Y_{T}^{(p)}\right]<\infty$ for any $p \geq 1$ by Lemma 8 . Let $\varepsilon \in(0,1 / 2)$. Note that $\log x<\varepsilon^{-1} x^{2 \varepsilon}$ for all $x>0$, so $\left(\delta^{2 \varepsilon} \log \left(4 E_{T} / \delta\right)\right)^{2} \leq \varepsilon^{-2}\left(4 E_{T}\right)^{4 \varepsilon}$. As the right hand side has finite expectation and is independent of $\delta$, by the dominated convergence theorem, $\delta^{2(2 \varepsilon-1)} \mathbb{E}\left[V_{1}^{2}\right]=16 C_{1}^{2} \mathbb{E}\left[\left(\delta^{2 \varepsilon} \log \left(4 E_{T} / \delta\right)\right)^{2}\right] \rightarrow 0$ as $\delta \downarrow 0$. Therefore, there exists $\delta_{0}=\delta_{0}(\varepsilon) \in(0,1)$ such that $\mathbb{E}\left[V_{1}^{2}\right]<\delta^{2(1-2 \varepsilon)}$ for all $\delta \in\left(0, \delta_{0}\right)$. We have thus obtained the inequality $\mathbb{E}\left[Z_{T}^{2}\right] \leq C_{3}\left(\delta^{1-2 \varepsilon}+\right.$ $\left.\delta^{2 \theta_{F}}+\delta^{2 \theta_{G}}\right)$ for some finite constant $C_{3}$. The obvious inequality $\mathbb{E}\left[Z_{T}\right] \leq\left(\mathbb{E}\left[Z_{T}^{2}\right]\right)^{1 / 2}$ now completes the proof of the theorem with condition (i).

We now turn to the proof of the theorem with condition (ii) that $G(t, x)=G(t)$ for $(t, x) \in[0, T] \times \mathbb{R}$. Since $G(t, x)$ does not depend on $x$, the integral $\int_{0}^{t} \mathbb{E}_{B}\left[Z_{r}^{2}\right] \mathrm{d} E_{r}$ in (32) vanishes. Moreover, since condition (12) simplifies to $|G(s)-G(t)| \leq K|s-t|^{\theta_{G}}$, the expression $\mathbb{E}_{B}\left[Y_{T}^{(2)}\right]$ in (32) also disappears. Thus, by inequalities (25), (28), (30), (32) and (34), as well as the obvious inequality $\delta \leq \delta^{\theta_{F}}$ valid for $\delta \in(0,1)$,

$$
\mathbb{E}_{B}\left[Z_{t}\right] \leq V_{3}+K \int_{0}^{t} \mathbb{E}_{B}\left[Z_{r}\right] \mathrm{d} E_{r}
$$

where

$$
V_{3}=K \delta^{\theta_{F}}\left(1+T^{\theta_{F}} E_{T}^{1-\theta_{F}}\right) \mathbb{E}_{B}\left[Y_{T}^{(1)}\right]+C_{4} \delta^{\theta_{G}} E_{T}^{1 / 2-\theta_{G}}+\left(C_{1} \delta \log \left(\frac{4 E_{T}}{\delta}\right)\right)^{1 / 2}
$$

with $C_{4}$ being a constant depending on $K, T, \theta_{G}$ and $b_{2}$. Applying a Gronwall-type inequality and taking $\mathbb{E}_{D}$ on both sides of the obtained inequality with $t=T$ gives

$$
\mathbb{E}\left[Z_{T}\right] \leq \mathbb{E}\left[V_{3} e^{K E_{T}}\right] \leq\left(\mathbb{E}\left[V_{3}^{2}\right] \cdot \mathbb{E}\left[e^{2 K E_{T}}\right]\right)^{1 / 2} .
$$

Note that since $\mathbb{E}\left[e^{2 K E_{T}}\right]<\infty$ for any inverse subordinator with underlying Lévy measure being infinite, we do not need to impose condition (i) that $\psi \in \mathrm{RV}_{\beta}$ with $\beta \in(1 / 2,1)$. The remainder of the proof is omitted since it is very similar to the argument given in the last part of the previous paragraph.

Remark 9. (1) Theorem 7 is still valid when the underlying Laplace exponent takes the form $\widehat{\psi}(s)=$ as $+\psi(s)$ with $a>0$ and $\psi \in \mathrm{RV}_{\beta}$ as in Remark 6(1).

(2) If $G(t, x)$ depends on $x$, then in the above proof the analysis of the squared error $Z_{t}^{2}$ instead of the $p$ th power error $Z_{t}^{p}$ with $p \neq 2$ is essential. Indeed, a straightforward modification of the proof would not lead to an inequality for $\mathbb{E}_{B}\left[Z_{t}^{p}\right]$ to which the Gronwall-type inequality is readily applicable.

This is because replacing the integral $\int_{0}^{t} \mathbb{E}_{B}\left[Z_{r}^{2}\right] \mathrm{d} E_{r}$ in (32) by the integral $\int_{0}^{t} \mathbb{E}_{B}\left[Z_{r}^{p}\right] \mathrm{d} E_{r}$ using the current method does not seem to be possible. Moreover, the appearance of the integral $\int_{0}^{t} \mathbb{E}_{B}\left[Z_{r}^{2}\right] \mathrm{d} E_{r}$ in (32) requires the estimate (29) for $I_{1}^{2}$ be used instead of the estimate (28) for $I_{1}$. The presence of $E_{T}$ in front of the integral $\int_{0}^{t} Z_{r}^{2} \mathrm{~d} E_{r}$ in (29) (and hence in (35) as well) is what amounts to the expression $\mathbb{E}\left[e^{16 K^{2}\left(E_{T}+b_{2}\right) E_{T}}\right]$. Condition (i) was imposed to guarantee the finiteness of the latter.

(3) The Euler-Maruyama scheme for classical Itô SDEs (without a random time change) has order $1 / 2$ of strong uniform convergence (see Theorem 10.2.2 of [16]). On the other hand, for SDE (8), it only seems possible to derive an order strictly less than $1 / 2$. This is because even in the simple case when $G(t, x) \equiv 1$, in order to control the quantity $I_{4}=\sup _{0 \leq s \leq t}\left|B_{E_{s}}-B_{E_{s}^{\delta}}\right|$, we need to use a result about the modulus of continuity for Brownian motion, which involves a logarithmic correction. However, the rate of convergence at the time horizon $T$ can be slightly improved since the discussion of the modulus of continuity is unnecessary. Namely, it follows that $\mathbb{E}\left[\left|X_{T}-X_{T}^{\delta}\right|\right] \leq C\left(\delta^{\theta_{F}}+\delta^{\theta_{G}}+\delta^{1 / 2}\right)$ for all $\delta \in(0,1)$.

Acknowledgements: The authors appreciate various comments and suggestions by anonymous referees that resulted in a succinct, better-organized paper with an improved result.

\section{References}

[1] D. A. Benson, S. W. Wheatcraft, and M. M. Meerschaert. Application of a fractional advectiondispersion equation. Water Resour. Res., 36(6):1403-1412, 2000. 
[2] N. H. Bingham, C. M. Goldie, and J. L. Teugels. Regular Variation. Encyclopedia of Mathematics and its Applications. Cambridge University Press, 1987.

[3] R. Cont and P. Tankov. Financial Modelling with Jump Processes. Chapman and Hall/CRC, 2003.

[4] M. Fischer and G. Nappo. On the moments of the modulus of continuity of Itô processes. Stoch. Anal. Appl., 28(1):103-122, 2008.

[5] R. N. Ghosh and W. W. Webb. Automated detection and tracking of individual and clustered cell surface low density lipoprotein receptor molecules. Biophys. J., 66(5):1301-1318, 1994.

[6] R. Gorenflo, F. Mainardi, E. Scalas, and M. Raberto. Fractional calculus and continuous-time finance III: the diffusion limit. Mathematical Finance, Trends in Mathematics, pages 171-180, 2001.

[7] M. Hahn, K. Kobayashi, J. Ryvkina, and S. Umarov. On time-changed Gaussian processes and their associated Fokker-Planck-Kolmogorov equations. Elect. Comm. in Probab., 16:150-164, 2011.

[8] M. Hahn, K. Kobayashi, and S. Umarov. Fokker-Planck-Kolmogorov equations associated with time-changed fractional Brownian motion. Proc. Amer. Math. Soc., 139(2):691-705, 2011.

[9] M. Hahn, K. Kobayashi, and S. Umarov. SDEs driven by a time-changed Lévy process and their associated time-fractional order pseudo-differential equations. J. Theoret. Probab., 25(1):262-279, 2012.

[10] E. Heinsalu, M. Patriarca, I. Goychuk, and P. Hänggi. Use and abuse of a fractional Fokker-Planck dynamics for time-dependent driving. Phys. Rev. Lett., 99:120602, 2007.

[11] J. Jacod. Calcul Stochastique et Problèmes de Martingales, volume 714 of Lecture Notes in Mathematics. Springer, Berlin, 1979.

[12] J. Jacod and A. N. Shiryaev. Limit Theorems for Stochastic Processes, volume 288 of Grundlehren der mathematischen Wissenschaften. Springer, Berlin, 2003.

[13] N. C. Jain and W. E. Pruitt. Lower tail probability estimates for subordinators and nondecreasing random walks. Ann. Probab., 15(1):75-101, 1987.

[14] E. Jum and K. Kobayashi. A strong and weak approximation scheme for stochastic differential equations driven by a time-changed Brownian motion. Probab. Math. Statist., 36(2):201-220, 2016.

[15] I. Karatzas and S. Shreve. Brownian Motion and Stochastic Calculus. Springer-Verlag New York, second edition, 1998.

[16] P. E. Kloeden and E. Platen. Numerical Solution of Stochastic Differential Equations. Springer, corrected edition, 1992.

[17] K. Kobayashi. Stochastic calculus for a time-changed semimartingale and the associated stochastic differential equations. J. Theoret. Probab., 24(3):789-820, 2011.

[18] L. Lv, W. Qiu, and F. Ren. Fractional Fokker-Planck equation with space and time dependent drift and diffusion. J. Stat. Phys., 149:619-628, 2012.

[19] M. Magdziarz. Langevin picture of subdiffusion with infinitely divisible waiting times. J. Stat. Phys., 135:763-772, 2009.

[20] M. Magdziarz. Stochastic representation of subdiffusion processes with time-dependent drift. Stoch. Proc. Appl., 119:3238-3252, 2009.

[21] M. Magdziarz, J. Gajda, and T. Zorawik. Comment on fractional Fokker-Planck equation with space and time dependent drift and diffusion. J. Stat. Phys., 154:1241-1250, 2014. 
[22] M. Magdziarz, S. Orzel, and A. Weron. Option pricing in subdiffusive Bachelier model. J. Stat. Phys., 145(1):187, 2011.

[23] M. Magdziarz and T. Zorawik. Stochastic representation of fractional subdiffusion equation. The case of infinitely divisible waiting times, Lévy noise and space-time-dependent coefficients. Proc. Amer. Math. Soc., 144:1767-1778, 2016.

[24] M. M. Meerschaert, E. Nane, and Y. Xiao. Correlated continuous time random walks. Statist. Probab. Lett., 79:1194-1202, 2009.

[25] M. M. Meerschaert and H-P. Scheffler. Limit theorems for continuous-time random walks with infinite mean waiting times. J. Appl. Probab., 41:623-638, 2004.

[26] M. M. Meerschaert and H-P. Scheffler. Triangular array limits for continuous time random walks. Stoch. Proc. Appl., 118:1606-1633, 2008.

[27] R. Metzler and J. Klafter. The random walk's guide to anomalous diffusion: a fractional dynamics approach. Phys. Rep., 339(1):1-77, 2000.

[28] E. Nane and Y. Ni. Stochastic solution of fractional Fokker-Planck equations with space-timedependent coefficients. J. Math. Anal. Appl., 442:103-116, 2016.

[29] E. Nane and Y. Ni. Stability of the solution of stochastic differential equation driven by timechanged Lévy noise. Proc. Amer. Math. Soc., 145:3085-3104, 2017.

[30] E. Nane and Y. Ni. Path stability of stochastic differential equations driven by time-changed Lévy noises. ALEA, Lat. Am. J. Probab. Math. Stat., 15:479-507, 2018.

[31] R. R. Nigmatullin. The realization of the generalized transfer equation in a medium with fractal geometry. Phys. Status Solidi B, 133:425-430, 1986.

[32] Ö. Önalan. Subdiffusive Ornstein-Uhlenbeck processes and applications to finance. Proceedings of the World Congress on Engineering 2015, II, 2015.

[33] P. Protter. Stochastic Integration and Differential Equations. Springer, second edition, 2004.

[34] K. Sato. Lévy Processes and Infinitely Divisible Distributions. Cambridge University Press, 1999.

[35] M. J. Saxton and K. Jacobson. Single-particle tracking: applications to membrane dynamics. Annu. Rev. Biophys. Biomol. Struct., 26:373-399, 1997.

[36] I. M. Sokolov and J. Klafter. Field-induced dispersion in subdiffusion. Phys. Rev. Lett., 97:140602, 2006.

[37] S. Umarov, M. Hahn, and K. Kobayashi. Beyond the Triangle: Brownian Motion, Itô Calculus, and Fokker-Planck Equation - Fractional Generalizations. World Scientific, 2018.

[38] A. Weron, M. Magdziarz, and K. Weron. Modeling of subdiffusion in space-time-dependent force fields beyond the fractional Fokker-Planck equation. Phys. Rev. E, 77:036704, 2008.

[39] Q. Wu. Stability of stochastic differential equation with respect to time-changed Brownian motion. Available at arXiv:1602.08160. 2016.

[40] G. M. Zaslavsky. Fractional kinetic equation for Hamiltonian chaos. Physica D, 76(1):110-122, 1994. 


\title{
Erratum to "Strong approximation of stochastic differential equations driven by a time-changed Brownian motion with time-space-dependent coefficients" [J. Math. Anal. Appl. 476(2) (2019), 619-636]
}

\author{
Sixian Jin* and Kei Kobayashi ${ }^{\dagger}$
}

In the first paragraph of the proof of [2, Theorem 1], L'Hospital's rule is used to obtain $\psi^{\prime} \in \mathrm{RV}_{\beta-1}$. However, that argument would work only if we a priori knew that the resulting limit existed. Since we are not sure whether the limit exists, we modify [2, Theorem 1] as follows.

- In the statement of the theorem, assume additionally that $\psi^{\prime} \in \mathrm{RV}_{-1}$ when $\beta=0$ (no additional assumptions are required when $\beta \in(0,1))$;

- In the proof, replace the first paragraph with the following:

First, suppose $\psi \in \mathrm{RV}_{\beta}$ with $\beta \in(0,1)$. Then due to the representation $\psi(s)=\int_{0}^{s} \psi^{\prime}(r) \mathrm{d} r$ and the fact that $\psi^{\prime}$ is monotone as $\psi^{\prime \prime}<0$, the monotone density theorem (see [1, Theorem 1.7.2]) yields $\psi^{\prime} \in \mathrm{RV}_{\beta-1}$. On the other hand, $\psi^{\prime} \in \mathrm{RV}_{-1}$ when $\beta=0$ by the additional assumption. (Note that the monotone density theorem does not give the same conclusion when $\beta=0$.) Next, regardless of the value of $\beta \in[0,1), \psi^{\prime}(\infty)=0$ by Lemma $4(1)$, so $\psi^{\prime}(s)=\int_{s}^{\infty}\left(-\psi^{\prime \prime}(r)\right) \mathrm{d} r$. Since $-\psi^{\prime \prime}$ is monotone as $\psi^{\prime \prime \prime}>0$, another version of the monotone density theorem (see the comment following the proof of $\left[1\right.$, Theorem 1.7.2]) yields $-\psi^{\prime \prime} \in \mathrm{RV}_{\beta-2}$.

Finally, note that the additional assumption that $\psi^{\prime} \in \mathrm{RV}_{-1}$ when $\beta=0$ neither excludes the important special case of the Gamma subordinator discussed in [2, Example 5(2)] nor affects the proofs given in the rest of the paper.

\section{References}

[1] N. H. Bingham, C. M. Goldie, and J. L. Teugels. Regular Variation. Encyclopedia of Mathematics and its Applications. Cambridge University Press, 1987.

[2] S. Jin and K. Kobayashi. Strong approximation of stochastic differential equations driven by a timechanged Brownian motion with time-space-dependent coefficients. J. Math. Anal. Appl., 476(2):619$636,2019$.

*Fordham University. Email: sjin27@fordham.edu

${ }^{\dagger}$ Corresponding author. Fordham University. Email: kkobayashi5@fordham.edu 